\title{
The Spinal Substrate of the Suppression of Action during Action Observation
}

\author{
Alexis V. Stamos, Helen E. Savaki, and Vassilis Raos \\ Department of Basic Sciences, Faculty of Medicine, School of Health Sciences, University of Crete, GR-71003 Iraklion, and Institute of Applied and \\ Computational Mathematics, Foundation for Research and Technology Hellas, GR-71110 Iraklion, Crete Greece
}

We have previously demonstrated that the forelimb representations of the primary motor and somatosensory cortices, as well as several premotor and parietal areas, are activated by both action-execution and action-observation, indicating that the spectator mentally simulates the observed action. Moreover, several studies demonstrated repeatedly that corticospinal excitability is modulated during action observation, providing evidence of an activation of the observer's motor system. However, evidence for the involvement of the spinal cord in action observation is controversial. The aim of the present study was to explore whether and how action-observation affects the spinal cord. To this end, we analyzed the spinal cord of eight monkeys (Macaca mulatta) trained to either execute reachingto-grasp movements or observe the experimenter performing the same movements. Observation of grasping induced a bilateral decrease of glucose consumption in the spinal forelimb representation, whereas execution of grasping induced an increase of glucose utilization in the same area, ipsilaterally to the grasping hand. The depression of overall activity in the cervical enlargement of the spinal cord for action-observation may explain the suppression of overt movements, despite the activation of the observer's motor system.

\section{Introduction}

Understanding what others do when we observe them acting is vital for our interaction with them. Recent imaging studies using the ${ }^{14} \mathrm{C}$-deoxyglucose $\left({ }^{14} \mathrm{C}\right.$-DG) method demonstrated that the areas activated in the brain of an actor performing a grasping movement and those of an observer watching the performance of the very same movement by another subject are virtually the same. This common neural network involves the forelimb representations of the primary motor and somatosensory cortices; several premotor and cingulate areas; extensive regions of the posterior, lateral, and medial parietal; and the intraparietal cortex (Raos et al., 2004, 2007; Evangeliou et al., 2009). These findings support the notion that we understand observed actions by mentally simulating them.

Transcranial magnetic-stimulation studies have consistently demonstrated that evoked potentials of the muscles involved in the execution of an action are facilitated when this action is observed (Fadiga et al., 1995; Strafella and Paus, 2000; Clark et al., 2003), providing evidence of an activation of the motor system during action-observation. This facilitation depends on the complexity of the observed task (Brighina et al., 2000), the agent to whom the observed action is attributed (Schütz-Bosbach et al., 2006), the orientation (Maeda et al., 2002), and the side of the

\footnotetext{
Received April 22, 2010; revised July 7, 2010; accepted July 8, 2010.

This work was supported by Grant 03ED803 from the Greek Secretariat of Research and Technology and FP6 Grant IST-027574 from the European Union. This study was in partial fulfillment of the requirements for awarding the Ph.D. degree in the Brain and Mind Sciences to Alexis V. Stamos (co-funded by the European Social Fund and National resources). We thank Maria Kefaloyianni for expert technical assistance and Mina N. Evangeliou for participating in training and experiments.

Correspondence should be addressed to Vassilis Raos, Department of Basic Sciences, Faculty of Medicine, School of Health Sciences, University of Crete, P.0. Box 2208, GR-71003, Iraklion, Crete, Greece. E-mail: vraos@med.uoc.gr. DOI:10.1523/JNEUROSCI.2067-10.2010

Copyright $\odot 2010$ the authors $\quad$ 0270-6474/10/3011605-07\$15.00/0
}

body one is observing (Aziz-Zadeh et al., 2002). Also, this facilitation is independent of the posture of the observed hand (Urgesi et al., 2006), is comparable for biomechanically possible and impossible movements (Romani et al., 2005), as well as for intransitive body movements and goal-directed actions (Cattaneo et al., 2009), and is modulated together with distinct phases of a grasping action (Gangitano et al., 2001, 2004). However, the facilitation of the motor-evoked potentials reflects the increased excitability at the cortical level. Whether the increased motor cortex excitability propagates downstream to influence the excitability of the spinal cord is a controversial issue (Baldissera et al., 2001; Patuzzo et al., 2003).

In the present study, we explored whether and how action observation affects the spinal cord. For this purpose, we used the ${ }^{14} \mathrm{C}$-DG method to obtain high-resolution functional images of the spinal cord of monkeys that either observed or executed the same grasping movements. We revealed that the cervical enlargement is suppressed bilaterally for action-observation and activated ipsilaterally to the grasping hand for action-execution. The suppression of metabolic activity evoked by action-observation specifically involves the lower cervical segments where motoneurons innervating the distal limb musculature reside (Jenny and Inukai, 1983; Chiken et al., 2001). Our study provides evidence for the existence of an inhibitory mechanism blocking, at the level of the spinal cord, the motor command issued in the observer's brain. A brief description of these results has appeared previously in abstract form (Stamos et al., 2009).

\section{Materials and Methods}

Subjects and tasks. Eight adult female Rhesus monkeys weighing between 4 and $5 \mathrm{~kg}$ were used. All animals were purpose-bred by authorized suppliers within the European Union (Deutches Primatenzentum and R.C. Hartelust BV) and were cared for in accordance with European 
Union (directive 86/609 and its amendments) and national regulations, as well as the National Institutes of Health's Principles of Laboratory Animal Care. Experimental protocols were approved by the institutional animal use committee. A detailed description of the surgical procedures, the recording of eye position, and EMG was reported previously (Raos et al., 2004, 2007; Evangeliou et al., 2009). All surgical procedures were performed under general anesthesia and aseptic conditions. To minimize pain or discomfort, analgesics and antibiotics were administered preoperatively and postoperatively. Animals were prepared for behavioral experiments by anchoring with acrylic cement a stainless-steel bolt onto mandibular plates secured on the cranium with titanium screws (Synthes) for head restraint. Eye position was recorded with an infrared oculometer (Dr. Bouis Devices) and electromyograms using $\mathrm{Ag}-\mathrm{AgCl}$ surface electrodes from the biceps and wrist extensor muscles (gain $\times 2000$, bandpass filter $0.3-3000 \mathrm{kHz}$ ). Eye and muscle signals were digitized at a rate of 500 and $1000 \mathrm{~Hz}$, respectively, using an A/D converter and stored on a hard disk for offline analysis. Training lasted for at least $1 \mathrm{~h}$ per day for 6-9 months until the monkeys perfected their performance ( $\sim 95 \%$ success rate). Successful completion of each trial was rewarded with water through a water delivery tube placed close to their mouth.

Behavioral tasks. The behavioral apparatus was placed in front of the monkeys at shoulder height, 20 or $50 \mathrm{~cm}$ away depending on whether the monkey or the experimenter had to perform the reaching-to-grasp movement. The opening of a shutter (circular, $8^{\circ}$ diameter) located at the front side of the apparatus provided access to a horizontally oriented ring that had to be grasped, either by the monkey or the experimenter, using the hook grip (insertion of the index finger into the ring with pronated hand).

Three grasping-execution (E) monkeys were trained to reach and grasp with the left forelimb whereas the right one was restricted. One of the monkeys performed the task under visual guidance and the other two in complete darkness. In the first case, the monkey was required to fixate the illuminated object for $0.7-1 \mathrm{~s}$, until a dimming of the light would signal reaching, grasping, and pulling the ring with the left forelimb while maintaining fixation. In the second case, a low-frequency auditory cue $(90 \mathrm{~Hz})$, delivered from a speaker placed $25 \mathrm{~cm}$ in front of the monkey in the median sagittal plane below the behavioral apparatus, instructed the monkey to look straight ahead toward the memorized location of the object for $0.7-1 \mathrm{~s}$, until a second high-frequency auditory cue $(180 \mathrm{~Hz})$ signaled the generation of the learned action while the monkey maintained its gaze straight ahead. The movement was usually completed within 500-600 ms. The E monkeys were allowed to move their eyes outside a circular window ( $8^{\circ}$ diameter) only during the intertrial intervals (ranging between 2 and $2.5 \mathrm{~s}$ ). The unaffected right side of the spinal cord of each E monkey (contralateral to the moving arm) was used as control for its affected ipsilateral left side.

Three grasping-observation $(\mathrm{O})$ monkeys were trained to maintain their gaze within the $8^{\circ}$-diameter circular window while observing the experimenter grasping the ring with the hook grip. The experimenter was standing on the right side of the monkey and performed the reaching-tograsp movements with her right hand. The monkey could see the hand approaching the object, the preshaping of the hand, the interaction of the hand with the object, and subsequently the object grasping and holding. Observation-task parameters were similar to the ones described for the execution task. During the training and the experiments on graspingobservation, both hands of the monkeys were restricted. These monkeys initially mastered the visually guided grasping-execution task and then switched to the grasping-observation task. To avoid possible influences of this earlier grasping training on the observation effects, one of these monkeys was trained to grasp with its left hand, the second one with its right hand, and the third one with both hands consecutively. This way, any side-to-side difference due to the earlier grasping training would be cancelled out when the average quantitative map of the three left sides was compared with the average quantitative map of the three right sides of the spinal cord.

Two arm-motion $(\mathrm{Cm})$ monkeys were used as control for the $\mathrm{O}$ monkeys. The Cm monkeys were trained to maintain their gaze straight ahead (within the $8^{\circ}$-diameter circular window) while the shutter of the behav- ioral apparatus opened and the object appeared and while the shutter closed and the experimenter approached it with one hand extended. Accordingly, the task of the $\mathrm{Cm}$ monkeys contained neither the hand preshaping nor its interaction with the object. During the training and experiments, both hands of the $\mathrm{Cm}$ monkeys were restricted. Intertrial intervals ranged between 2 and $2.5 \mathrm{~s}$.

${ }^{14} \mathrm{C}$-DG experiments. The ${ }^{14} \mathrm{C}-\mathrm{DG}$ experiment and the brain tissue processing for autoradiography were performed as previously described (Savaki et al., 1993, 1997). On the experimental day, monkeys were catheterized through femoral vein and artery under general anesthesia and were allowed $4-5 \mathrm{~h}$ to recover. Plasma glucose levels, blood pressure, hematocrit, and blood gases ranged within normal values in all monkeys and remained constant throughout the ${ }^{14} \mathrm{C}-\mathrm{DG}$ experiment. The measurement of local cerebral glucose utilization (LCGU) was initiated by the intravenous injection of $\left[{ }^{14} \mathrm{C}\right]$ deoxyglucose as a pulse of $100 \mu \mathrm{Ci} / \mathrm{kg}$ 2-deoxy-D- $\left[1-{ }^{14} \mathrm{C}\right]$ glucose (specific activity, $55 \mathrm{mCi} / \mathrm{mmol}$; ARC) dissolved in saline delivered $5 \mathrm{~min}$ after each monkey started its behavioral task. Timed arterial samples were collected from the catheterized femoral artery over the next $45 \mathrm{~min}$ at a predetermined schedule. Plasma ${ }^{14} \mathrm{C}-\mathrm{DG}$ and glucose concentrations were measured. At $45 \mathrm{~min}$ after the ${ }^{14} \mathrm{C}-\mathrm{DG}$ administration, the monkey was killed by intravenous injections of $50 \mathrm{mg}$ of sodium thiopental in $5 \mathrm{ml}$ of saline followed by a saturated potassium chloride solution to stop the heart. The cerebral hemispheres, the cerebellum, and the spinal cord were removed, frozen in isopentane at $-50^{\circ} \mathrm{C}$ and stored at $-80^{\circ} \mathrm{C}$ until sectioning for autoradiography. Serial $20-\mu \mathrm{m}$-thick horizontal sections were cut in a cryostat at $-20^{\circ} \mathrm{C}$. Autoradiographs were prepared by exposing these sections, together with precalibrated ${ }^{14} \mathrm{C}$-standards, on medical x-ray film (Kodak Biomax MR) in $\mathrm{x}$-ray cassettes. Demarcation of the spinal segments was based on the dorsal roots. Quantitative densitometric analysis of autoradiographs was performed with a computerized image processing system (Imaging Research). The metabolic activity in LCGU values (in $\mu \mathrm{mol} / 100 \mathrm{~g} / \mathrm{min}$ ) was calculated from the original operational equation of the method (Sokoloff et al., 1977) using the appropriate kinetic constants for the monkey (Kennedy et al., 1978).

Reconstruction of quantitative spinal maps and their geometrical normalization. We generated two-dimensional (2D) reconstructions (glucograms) of the spatio-intensive pattern of metabolic activity in LCGU values within the rostrocaudal (from the first cervical to the first thoracic segments) and the dorsoventral (from the dorsal to the ventral horn) extent of the spinal gray matter, separately for the left and the right side. Each section contributed a data array (sampling resolution, $50 \mu \mathrm{m} /$ pixel) that was aligned with the arrays obtained from adjacent sections, for a total of $\sim 200$ serial sections of $20 \mu \mathrm{m}$ thickness for each spinal cord. In the illustrated average 2D-maps, the spatial resolution in both the rostrocaudal and dorsoventral dimensions is $100 \mu \mathrm{m}$. The alignment of the data arrays was based on marks on the white matter demarcating the spinal segments, made during the dissection of the spinal cords. The size of the spinal cord varied from animal to animal. To compensate for this variability, individual glucograms were geometrically normalized along with the spinal demarcation points, thus allowing for the direct comparison of the different maps. For this reason, averages of the lengths and heights of the spinal segments were separately estimated from all spinal cords to construct a reference map. The glucogram of each side of each spinal cord was then fit to this reference map using linear transformations of the plane (Moschovakis et al., 2001) with the help of Transform (Fortner Software) and custom-designed routines in the Matlab environment (Mathworks). With this procedure, we created geometrically normalized maps containing a standard number of pixels. Data from different geometrically normalized maps were combined to obtain the average-LCGU maps we illustrate. To generate average maps, the LCGU value found in a certain pixel in one of the geometrically normalized maps was added to the value found in the pixel occupying the same position in one or more other similar maps and the result was divided by the number of maps used.

Statistical analysis. Normalization of LCGU values was based on the averaged unaffected area of the spinal gray-matter value pooled across all monkeys (Raos et al., 2004, 2007; Evangeliou et al., 2009). To normalize metabolic activity, LCGU values were multiplied with a factor that was 

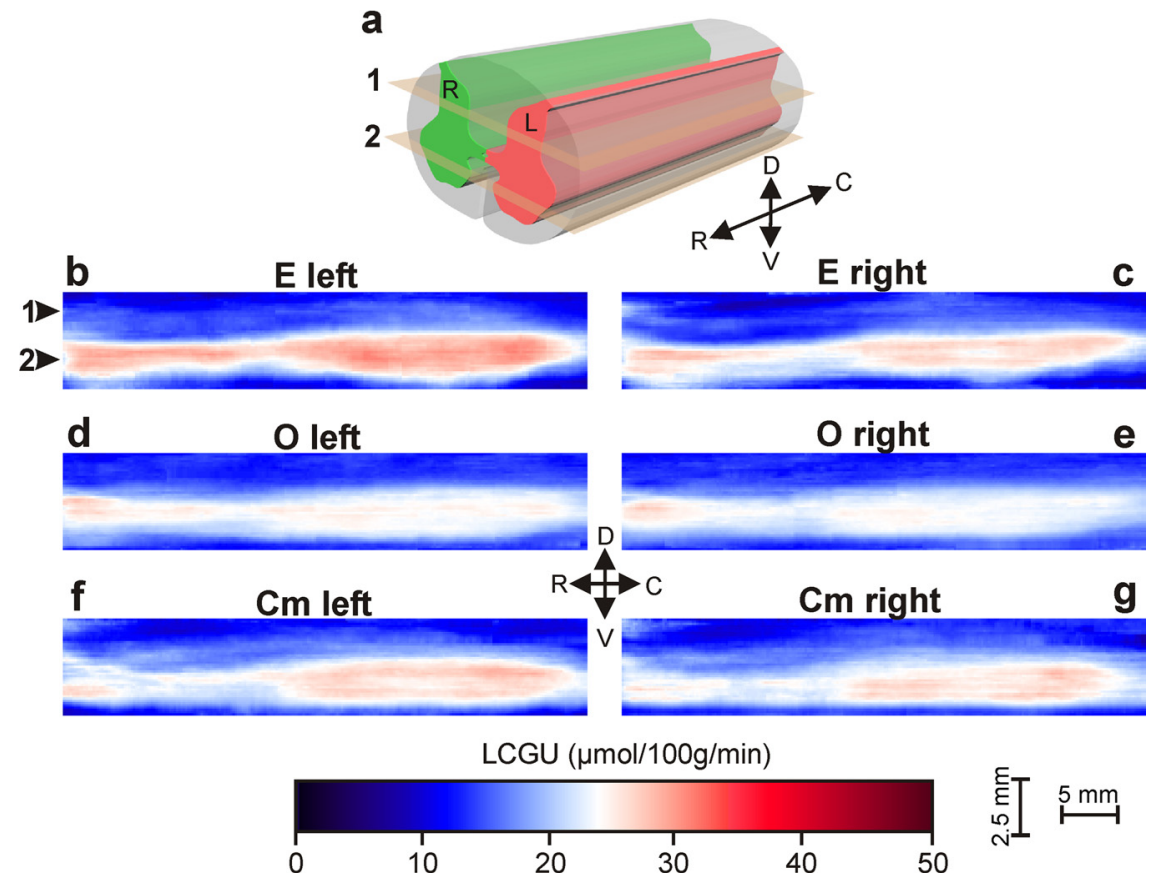

Figure 1. Metabolic effects induced by execution and observation of grasping movements in the cervical and first thoracic segments of the spinal cord. Each color-coded map represents the average of the geometrically normalized, quantitative maps (glucograms) in each side of the spinal cord. $\boldsymbol{a}$, Three-dimensional schematic representation of the spinal cord (anterolateral view). The spinal gray matter in the right and the left side of the spinal cord is illustrated by the green- and the red-painted volumes, respectively. Horizontal planes 1 and 2 correspond to two different dorsoventral levels of sectioning, traversing the spinal cord through the dorsal and ventral horns, respectively. $\boldsymbol{b}, \boldsymbol{c}$, Average spinal maps in the left $(\boldsymbol{b})$ and right (c) sides of the three monkeys executing grasping movements with their left forelimb (E). Arrows 1 and 2 indicate the dorsoventral levels of the corresponding planes in $\boldsymbol{a}$. A pronounced activation in the intermediate zone and the ventral horn is apparent in the side ipsilateral to the grasping forelimb (Eleft) compared with the contralateral side (E right). $\boldsymbol{d}, \boldsymbol{e}$, Average spinal maps in the left ( $\boldsymbol{d}$ ) and the right $(\boldsymbol{e})$ sides of the three monkeys observing grasping movements executed with the right hand of the experimenter $(0)$. A significant suppression in the intermediate zone and the ventral horn in both 0 left and 0 right is apparent, compared with the arm-motion control monkeys $(\mathrm{Cm})$ and with the control side of the executing monkeys (E right). $\boldsymbol{f}, \boldsymbol{g}$, Average spinal maps in the left $(\boldsymbol{f})$ and right $(\boldsymbol{g})$ sides of the two Cm monkeys. Color bar represents the LCGU values in $\mu \mathrm{mol} / 100 \mathrm{~g} / \mathrm{min}$. C, Caudal; D, dorsal; $R$, rostral: $V$, ventral.

separately determined for each spinal cord. This factor is equal to the ratio of the mean LCGU value found in an unaffected spinal gray-matter region of the spinal cord in question over the mean LCGU value obtained from the same region after pooling all spinal cords. Percentage LCGU differences between the affected left (ipsilateral to the moving hand) and the control right (contralateral to the moving hand) side of the spinal cord of the E monkeys were calculated as follows: (Eleft - Eright)/Eright $\times 100$. Percentage LCGU differences between the $\mathrm{O}$ and the $\mathrm{Cm}$ monkeys were calculated as follows: $(\mathrm{O}-\mathrm{Cm}) / \mathrm{Cm} \times 100$. Because side-to-side differences in normal monkeys rise up to $7 \%$ (Kennedy et al., 1978), only differences $>7 \%$ were considered for statistical treatment. To determine statistical significant differences, we relied on Student's unpaired $t$ test (Raos et al., 2004, 2007; Evangeliou et al., 2009).

\section{Results}

On the day of the ${ }^{14} \mathrm{C}-\mathrm{DG}$ experiment, all monkeys executed their tasks for the entire experimental period (45 min). Success rate remained approximately the same $(>90 \%)$ throughout the experiment. Since the concentration of ${ }^{14} \mathrm{C}-\mathrm{DG}$ in the blood decays with a time constant equal to $\sim 4 \mathrm{~min}$, only $15 \%$ of the tracer is available after the 10 first minutes of the experiment, and therefore the monkeys' behavior after this initial period influences LCGU values insignificantly (Sokoloff et al., 1977). For this reason, we provide quantitative description of the behavior of the monkeys during the critical period of the first $10 \mathrm{~min}$. The oculomotor performance of some of the monkeys, either as instantaneous eye position averaged over all trials or as three- dimensional histograms of the dwell time of the line of sight as a function of eye position, during the critical 10 first minutes of the ${ }^{14} \mathrm{C}-\mathrm{DG}$ experiment was presented previously (Raos et al., 2007; Evangeliou et al., 2009). Presently, the oculomotor and skeletomotor performance are briefly reported. Because behavioral performance may influence the LCGU values, the mean rate of movements for the execution, the observation, and the arm-motion tasks were set to be similar.

The three E monkeys executed an average of 11 arm movements per minute and kept their eyes within the window of the behavioral apparatus for $7 \mathrm{~min}$ during the critical 10 first minutes of the ${ }^{14} \mathrm{C}-\mathrm{DG}$ experiment. We generated glucograms of the E monkeys by averaging the three geometrically normalized quantitative spinal maps of metabolic activity in each side of the spinal cord, separately. When the average spinal map of the left side (Fig. $1 b$ ) is compared with the corresponding map of the right side (Fig. 1c), increased metabolic activity is apparent in the intermediate zone and the ventral horn (lower half of the map) of the left side, ipsilateral to the moving hand, as expected (Table 1). Electromyographic activity recorded from the biceps and wrist extensor was increased in the left forelimb executing the movements, whereas it was at baseline levels in the right nonperforming forelimb (Fig. 2).

The three $\mathrm{O}$ monkeys observed an average of 12 arm movements per minute and fixated within the window of the behavioral apparatus for 7 min during the critical 10 first minutes of the ${ }^{14} \mathrm{C}-\mathrm{DG}$ experiment. When the average spinal map of the left side (Fig. 1d) is compared with the average spinal-map of the right side (Fig. 1e), no side-to-side difference is apparent (Table 1 ). Both sides of the $\mathrm{O}$ monkeys (Fig. 1d,e) display decreased metabolic activity in the lower cervical segment when compared, not only with the map of the activated left side (Fig. $1 b$ ), but also with the map of the control right side of the E monkeys (Fig. 1c). Electromyographic activity in the biceps and wrist extensor was unaffected in both forelimbs of the monkeys observing grasping movements, same way as in the nonperforming forelimb of the monkeys executing grasping movements (Fig. 2).

The Cm monkeys were used to exclude potential effects induced by the observation of the objects, the eye movements while scanning these objects, and the biological movement of the experimenter's arm. The difference between the $\mathrm{O}$ and the $\mathrm{Cm}$ monkeys is that, unlike the former, the $\mathrm{Cm}$ monkeys observed neither the preshaping of the approaching hand nor the interaction of the hand with the object. The two Cm monkeys observed an average of 12 movements per minute and maintained their gaze within the window of the behavioral apparatus for $7 \mathrm{~min}$ during the critical 10 first minutes of the ${ }^{14} \mathrm{C}-\mathrm{DG}$ experiment. When the average spinal map of the left side (Fig. $1 f$ ) is compared with the average spinal map of the right side (Fig. $1 g$ ), no side-toside difference is apparent (Table 1). The metabolic activity in 
Table 1. Metabolic effects in the spinal cord of the monkey brain

\begin{tabular}{|c|c|c|c|c|c|c|c|c|c|c|}
\hline $\begin{array}{l}\text { Intermediate zone and ventral } \\
\text { horn rostrocaudal extent }\end{array}$ & $\begin{array}{l}\mathrm{Cml} \\
(\mathrm{LCGU} \pm \mathrm{SD})\end{array}$ & $\begin{array}{l}\mathrm{Cmr} \\
(\mathrm{LCGU} \pm \mathrm{SD})\end{array}$ & $\mathrm{Cm}$ & $\begin{array}{l}\mathrm{El} \\
(\mathrm{LCGU} \pm \mathrm{SD})\end{array}$ & $\begin{array}{l}\mathrm{Er} \\
(\mathrm{LCGU} \pm \mathrm{SD})\end{array}$ & $\begin{array}{l}\text { Ol } \\
(\mathrm{LCGU} \pm \mathrm{SD})\end{array}$ & $\begin{array}{l}\text { Or } \\
(\mathrm{LCGU} \pm \mathrm{SD})\end{array}$ & 0 & $\mathrm{Er} / \mathrm{El}(\%)$ & $0 / \mathrm{Cm}(\%)$ \\
\hline 1st quarter & $23.0 \pm 1$ & $23.5 \pm 1$ & 23.3 & $26.5 \pm 2$ & $24.5 \pm 2$ & $24.0 \pm 1$ & $23.5 \pm 1$ & 23.8 & 8 & 2 \\
\hline 2nd quarter & $23.5 \pm 1$ & $23.5 \pm 1$ & 23.5 & $26.0 \pm 2$ & $24.0 \pm 1$ & $23.5 \pm 1$ & $23.0 \pm 1$ & 23.3 & 8 & -1 \\
\hline 3rd quarter & $25.0 \pm 1$ & $25.0 \pm 1$ & 25.0 & $27.5 \pm 2$ & $25.0 \pm 1$ & $23.5 \pm 1$ & $23.5 \pm 1$ & 23.5 & 10 & -6 \\
\hline 4th quarter & $25.5 \pm 2$ & $25.0 \pm 1$ & 25.3 & $27.5 \pm 2$ & $25.0 \pm 1$ & $23.5 \pm 1$ & $23.0 \pm 1$ & 23.3 & 10 & -8 \\
\hline
\end{tabular}

$\mathrm{Cml}$ and $\mathrm{Cmr}$ values represent the average LCGU values (in $\mu \mathrm{mol} / 100 \mathrm{~g} / \mathrm{min}$ ) from the two left and the two right sides of the spinal gray matter of the motion- control monkeys, respectively. ( $\mathrm{m}$ is the average of the $\mathrm{Cml}$ and $\mathrm{Cmr}$. El and Er values represent the average LCGU values from the three left and the three right sides of the spinal gray matter of the grasping-execution monkeys, respectively. 0 l and Or values represent the average $L C G U$ values from the three left and the three right sides of the spinal gray matter of the grasping- observation monkeys, respectively. 0 is the average of $0 \mathrm{l}$ and $\mathrm{Or}$. El/Er and $0 / \mathrm{Cm}$ values represent the percentage differences between El and Er and between 0 and $\mathrm{Cm}$ respectively, calculated as (experimental - control)/control $\times 100$. Values in bold indicate statistically significant differences by the Student's unpaired $t$ test at the level of $p<0.001$.

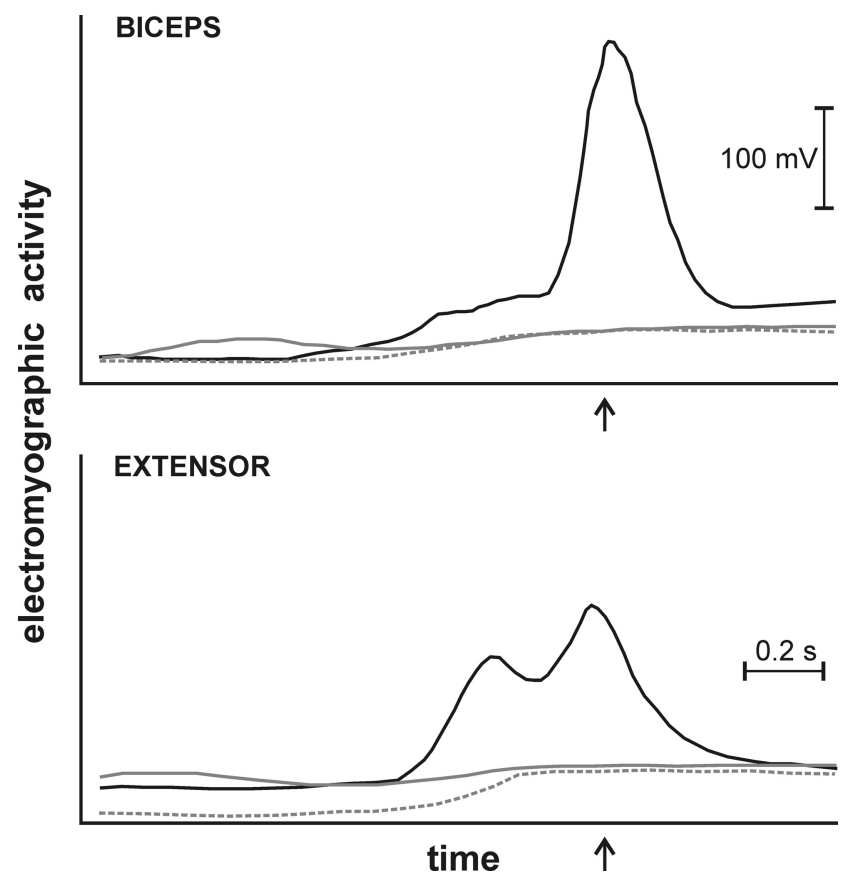

Figure 2. Averaged rectified electromyographic records from the biceps and wrist extensor The electromyographic activity recorded from the muscles of the performing (left) and nonperforming (right) forelimbs during action execution is represented by the solid black and dashed gray lines, respectively. The activity during action observation is represented by the solid gray lines. The records are aligned on the onset of movement (arrows). The activity recorded from the forelimb muscles of the observing monkeys was at baseline levels, same way as that recorded from the muscles of the nonperforming forelimb of the monkeys executing grasping movements. In contrast, activity recorded from the performing forelimb of the monkeys executing grasping movements was markedly enhanced.

both sides of the Cm monkeys (Fig. $1 f, g$ ) is similar to that in the control right side of the E monkeys (Fig. 1c) and higher than that in the spinal maps of the $\mathrm{O}$ monkeys (Fig. 1d,e; Table 1).

To illustrate graphically the spatial distribution of metabolic activity, we plotted the LCGU values in the intermediate zone and the ventral horn (Fig. 3). Each plot represents the average LCGU values and 95\% confidence intervals (per $100 \mu \mathrm{m}$ length) along the rostrocaudal extent of the spinal cord (from the first cervical to the first thoracic spinal segment). Because the left-to-right LCGU values along this rostrocaudal extent in the $\mathrm{Cm}$ and $\mathrm{O}$ monkeys did not differ significantly (Table 1), we averaged the left and the right sides of each one of these groups to obtain single graphs for the $\mathrm{Cm}$ and $\mathrm{O}$ monkeys. The plots manifest the following: (1) the activity in the right, control, spinal side of the grasping monkeys (Fig. 3, Er) is similar to that in the Cm monkeys (Fig. 3, Cm); (2) the activity in the intermediate zone and the ventral horn of the left spinal side (Fig. 3, El), ipsilateral to the moving forelimb, of the monkeys executing grasping movements

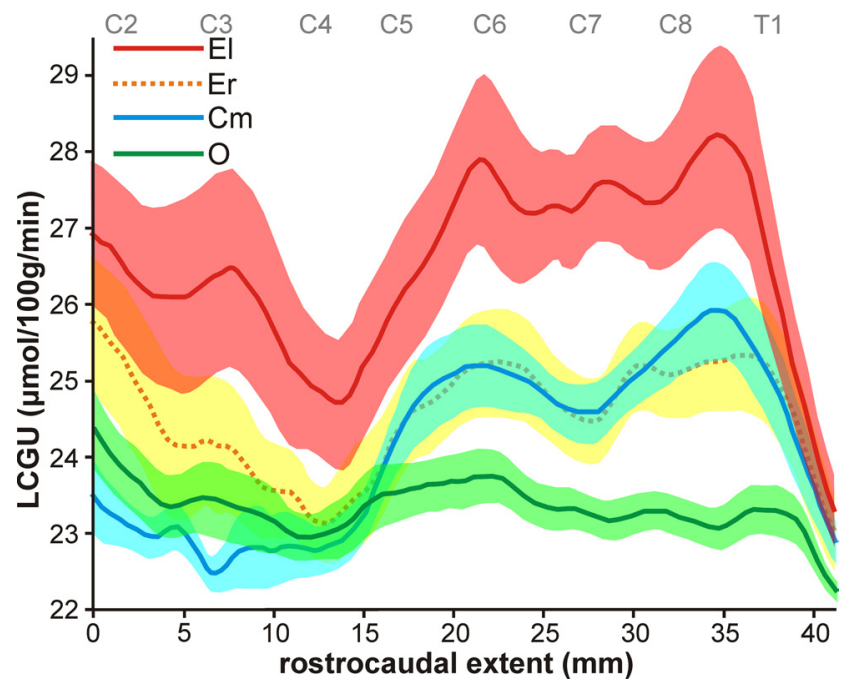

Figure 3. Plot of the LCGU values in the intermediate zone and the ventral horn of the spinal cord. Each plot represents average $\mathrm{LCGU}$ values $(\mu \mathrm{mol} / 100 \mathrm{~g} / \mathrm{min}$ ) and $95 \%$ confidence intervals per $100 \mu \mathrm{m}$, along the cervical and first thoracic segments of the spinal cord. Averages in the left (El) and the right (Er) sides of the three monkeys executing grasping movements with their left forelimb are represented by the solid red and dotted orange lines, respectively. The average in both sides, pooled together, of the three monkeys observing grasping movements executed with the right hand of the experimenter $(0)$ is represented by the green line. The average in both sides, pooled together, of the two arm-motion control monkeys $(\mathrm{Cm})$ is represented by the blue line.

is increased along the entire rostrocaudal extent; and (3) the activity in the intermediate zone and the ventral horn of both spinal sides in the monkeys observing the same grasping movements performed by the experimenter (Fig. 3, O) is suppressed along the lower segments of the cervical enlargement.

To graphically represent these differential effects, the percent LCGU differences in the intermediate zone and the ventral horn have been plotted along the cervical and first thoracic segments, for the El values in reference to the Er values, and for the $\mathrm{O}$ values in reference to the $\mathrm{Cm}$ values (Fig. 4). The activation induced by grasping-execution is evident along the entire examined anteroposterior extent of the spinal cord (Fig. 4, El/Er). In contrast, the suppression induced by grasping-observation is more prominent along the lower cervical and first thoracic segments (Fig. 4, $\mathrm{O} / \mathrm{Cm}$ ), where the motoneurons controlling the distal musculature of the forelimb are located (Jenny and Inukai, 1983; Chiken et al., 2001).

\section{Discussion}

In the present study, we revealed that the metabolic activity in the cervical enlargement of the spinal cord is suppressed bilaterally in monkeys observing reaching-to-grasp movements, whereas it is activated ipsilaterally to the grasping hand in monkeys executing the 


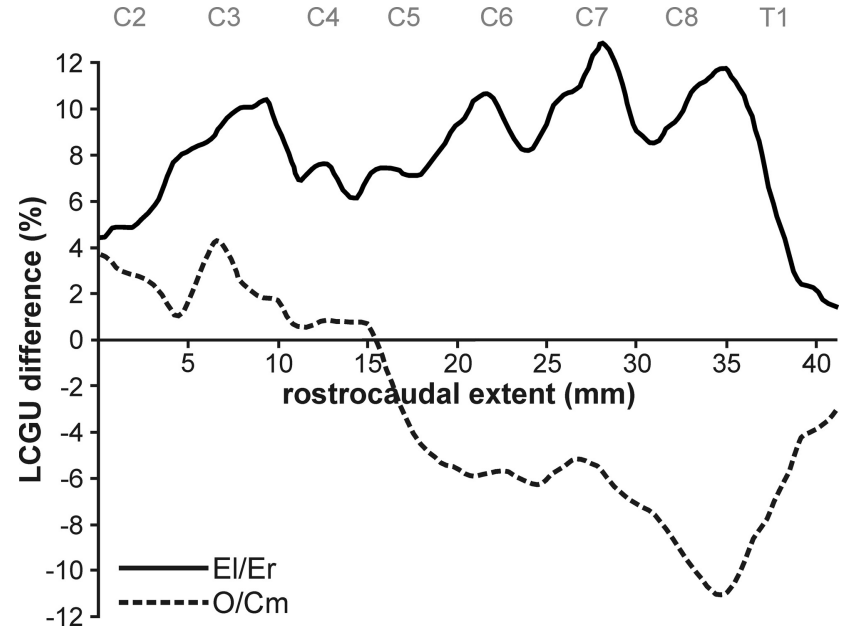

Figure 4. Plots of the percentage LCGU differences along the cervical and first thoracic segments of the intermediate zone and the ventral horn of the spinal cord. The solid line illustrates the differences in the intermediate zone and the ventral horn between the left and the right side in the three execution monkeys (E//Er). The dashed line illustrates the differences between the three observation monkeys and the arm-motion control $(0 / \mathrm{Cm})$. Baseline corresponds to $0 \%$ LCGU difference from the reference. The intermediate zone and the ventral horn of the left side are activated along all cervical and thoracic segments in the monkeys executing grasping movements. In contrast, the intermediate zone and the ventral horn are suppressed along the lower cervical and thoracic segments in the monkeys observing the same grasping movements performed by the experimenter.

same movements. The localization of the observation-induced spinal effect in the lower cervical and first thoracic segments, where the motoneurons innervating the distal musculature of the forelimb reside (Jenny and Inukai, 1983; Chiken et al., 2001), matches the localization of the observation-induced effect in the anterior bank of the central sulcus where the distal forelimb is represented within the primary motor cortex (Raos et al., 2007). Moreover, the observation-induced bilateral effects in the spinal cord parallel the bilateral effects in motor and premotor cerebral cortical areas elicited by action-observation (Raos et al., 2007). The suppression of spinal activity during action-observation may explain the absence of overt movements as well as the absence of muscle activation detected by EMG (Raos et al., 2004). It thus explains why actions are not elicited by the spectator, upon observing their execution by another subject, even though the spectator's primary motor cortex is activated (Raos et al., 2004, 2007).

Transcranial magnetic stimulation studies have demonstrated repeatedly that corticospinal excitability, as estimated from the amplitude of motor-evoked potentials, is enhanced by mental simulation of a movement during both action observation (Fadiga et al., 1995; Clark et al., 2003; Patuzzo et al., 2003; Borroni et al., 2005; Montagna et al., 2005; Léonard and Tremblay, 2007) and motor imagery (Izumi et al., 1995; Abbruzzese et al., 1996; Yahagi et al., 1996; Kasai et al., 1997; Kiers et al., 1997; Yahagi and Kasai, 1998; Fadiga et al., 1999; Rossini et al., 1999; Facchini et al., 2002; Clark et al., 2003; Patuzzo et al., 2003; Léonard and Tremblay, 2007). However, the enhanced amplitude of motor-evoked potentials provides information about the contribution of the motor cortex in the corticospinal excitability, rather than about the modulation of activity within the spinal cord. Studies aiming to evaluate the modulation of activity in the spinal cord during mental simulation provided conflicting results. Some researchers found no effect of mental simulation on the spinal cord (Abbruzzese et al., 1996; Yahagi et al., 1996; Kasai et al., 1997; Hashimoto and Rothwell, 1999; Facchini et al., 2002; Patuzzo et al.,
2003), whereas others reported a modulation (Kiers et al., 1997; Rossini et al., 1999; Baldissera et al., 2001; Li et al., 2004; Borroni et al., 2005, 2008; Montagna et al., 2005; Borroni and Baldissera, 2008). Indeed, the majority of the studies reporting spinal modulation during action-observation and motor-imagery concluded that the changes in the amplitude of the motor-evoked potentials are mainly due to excitability changes in the motor cortex (Kiers et al., 1997; Rossini et al., 1999; Borroni et al., 2005; Montagna et al., 2005; Borroni and Baldissera, 2008), whereas only one provided evidence for changes in the excitability of the spinal segmental circuitry, independent of the modulation of the motor cortex (Li et al., 2004).

Our study provides evidence for the involvement of the spinal cord in action-observation. The fact that the spinal segments of the forelimb representation were activated for action-execution and depressed for action-observation, although the forelimb representation of the primary motor cortex (MI/FI) was activated in both cases (Raos et al., 2004, 2007), indicates that our results in the spinal cord reflect not only the MI-corticospinal terminal activation but also the local interneuronal activity modulated by spinal input(s) additional to that originating in MI/FI. Should the spinal effects be nothing more than a by-product of incoming signals, either excitatory or inhibitory cortical descending input would result in elevated glucose consumption because both processes are energy consuming (Savaki et al., 1992). Thus, the inhibition of tonically active spinal interneurons most probably underlies the metabolic depression we detect in the spinal cord. More detailed, our previous ${ }^{14} \mathrm{C}$-DG studies demonstrated that the MI/F1-forelimb representation in the anterior bank of the central sulcus was activated for both action-execution and action-observation, with the latter eliciting smaller effect than the former (Raos et al., 2004). In contrast, premotor cortical area F5 was more activated for action-observation than for action-execution and premotor area F7 was activated only for actionobservation (Raos et al., 2007). Premotor cortical areas can influence spinal activity either through the primary motor cortex (Dum and Strick, 2005) or through their corticospinal (Dum and Strick, 1991; He et al., 1993) and cortico-brainstem-spinal projections (Kuypers, 1981; Keizer and Kuypers, 1989). The reticulospinal tract, which has long been considered to control motoneurons innervating axial and proximal muscles (Kuypers, 1981), has been recently demonstrated to also influence motoneurons projecting to distal limb muscles, including intrinsic hand muscles, and thus forming a pathway parallel to the corresponding corticospinal tract (Riddle et al., 2009). Premotor cortical areas have proven to facilitate the primary motor cortex (Shimazu et al., 2004; Schmidlin et al., 2008) and inhibit the spinal cord (Moll and Kuypers, 1977; Sawaguchi et al., 1996). Therefore, we propose that the premotor cortical areas F5 and F7, which were more affected by action-observation than by actionexecution (Raos et al., 2007), may provide an inhibitory input to the spinal cord of the observing monkeys, which suppresses the metabolic activity at the level of spinal interneurons. In parallel, the primary motor cortex, which was less affected by observation than by execution (Raos et al., 2004), may provide an excitatory input to the spinal cord, although smaller than that induced by execution of the same action. Our suggestion about an MI/FIinduced activation and a premotor-induced inhibition at the level of the spinal cord is in agreement with the dual mechanism, which was previously proposed to operate during action-simulation and which involves an excitatory component activating the motor system and a parallel inhibitory constituent suppressing the overt movement (Jeannerod, 2001). More specifically, area 
F7, which has been found activated for grasping-observation but not for grasping-execution (Raos et al., 2007), is able to mediate the depressed activity in the spinal cord during action-observation through its cortico-brainstem-spinal projections (Keizer and Kuypers, 1989). Alternatively and/or additionally, the increased activity that we have reported in area F5 of the observing monkeys compared with the executing subjects (Raos et al., 2007) could contribute to the metabolic depression that we measured in the spinal cord of the observing monkeys in the present study. Indeed, it has been recently demonstrated that area F5 is endowed with pyramidal tract neurons displaying high spontaneous activity, which is completely suppressed during action-observation. These neurons were reported to disfacilitate the spinal motoneurons (Kraskov et al., 2009). Moreover, it is known that the ability of descending projections to drive motoneurons may be considerably influenced by alterations in the activity of their target interneurons (Kuypers, 1981), which display high levels of spontaneous activity (Maier et al., 1998; Prut and Perlmutter, 2003). Thus, excitatory input to the spinal interneurons would increase their firing rate compared with the resting state, whereas inhibitory input would decrease their firing rate. Accordingly, in the case of action-execution, the increased metabolism measured in the forelimb representation of the spinal cord may reflect the sum of glucose consumption due to the activated corticospinal terminals and the excited spinal interneurons. Moreover, in the case of action-observation, the decreased glucose consumption may reflect the sum of the elevated activity due to the corticospinal terminals and the reduced activity due to the inhibited interneurons via premotor cortical input. Actually, the inhibition of a significant proportion of spinal interneurons while movements are withheld (Prut and Fetz, 1999) as well as their powerful inhibitory impact at the spinal population level (Asher et al., 2010) have been reported in the past.

Evidence for the existence of an inhibitory mechanism acting at the spinal level to prevent overt movements during action observation has been provided in the past. Baldissera and colleagues (2001) reported that the modulation pattern of the H-reflex during action-observation was opposite to that occurring during the actual execution of the observed action, and proposed that this finding might reflect the mechanism preventing the overt replica of the seen action (Baldissera et al., 2001). However, subsequent studies reported that the modulation pattern of the H-reflex and the facilitated motor-evoked potentials elicited by observation matches the temporal pattern of the muscle recruitment during action-execution, thus undermining the results and the interpretation of the earlier study (Borroni et al., 2005; Montagna et al., 2005; Borroni and Baldissera, 2008).

In conclusion, here we demonstrate for the first time the depression of overall activity in the spinal segments of forelimb representation during observation of reaching-to-grasp movements. This finding explains the suppression of overt movement during action-observation, despite the activation of the primary motor cortex in the observer's brain (Raos et al., 2004, 2007).

\section{References}

Abbruzzese G, Trompetto C, Schieppati M (1996) The excitability of the human motor cortex increases during execution and mental imagination of sequential but not repetitive finger movements. Exp Brain Res 111:465-472.

Asher I, Zinger N, Yanai Y, Israel Z, Prut Y (2010) Population-based corticospinal interactions in macaques are correlated with visuomotor processing. Cereb Cortex 20:241-252.

Aziz-Zadeh L, Maeda F, Zaidel E, Mazziotta J, Iacoboni M (2002) Lateral- ization in motor facilitation during action observation: a TMS study. Exp Brain Res 144:127-131.

Baldissera F, Cavallari P, Craighero L, Fadiga L (2001) Modulation of spinal excitability during observation of hand actions in humans. Eur J Neurosci 13:190-194.

Borroni P, Baldissera F (2008) Activation of motor pathways during observation and execution of hand movements. Soc Neurosci 3:276-288.

Borroni P, Montagna M, Cerri G, Baldissera F (2005) Cyclic time course of motor excitability modulation during the observation of a cyclic hand movement. Brain Res 1065:115-124.

Borroni P, Montagna M, Cerri G, Baldissera F (2008) Bilateral motor resonance evoked by observation of a one-hand movement: role of the primary motor cortex. Eur J Neurosci 28:1427-1435.

Brighina F, La Bua V, Oliveri M, Piazza A, Fierro B (2000) Magnetic stimulation study during observation of motor tasks. J Neurol Sci 174:122-126.

Cattaneo L, Caruana F, Jezzini A, Rizzolatti G (2009) Representation of goal and movements without overt motor behavior in the human motor cortex: a transcranial magnetic stimulation study. J Neurosci 29:11134-11138.

Chiken S, Hatanaka N, Tokuno H (2001) Distribution of median, ulnar and radial motoneurons in the monkey spinal cord: a retrograde triplelabeling study. Neurosci Lett 307:143-146.

Clark S, Tremblay F, Ste-Marie D (2004) Differential modulation of corticospinal excitability during observation, mental imagery and imitation of hand actions. Neuropsychologia 42:105-112.

Dum RP, Strick PL (1991) The origin of corticospinal projections from the premotor areas in the frontal lobe. J Neurosci 11:667-689.

Dum RP, Strick PL (2005) Frontal lobe inputs to the digit representations of the motor areas on the lateral surface of the hemisphere. J Neurosci 25:1375-1386.

Evangeliou MN, Raos V, Galletti C, Savaki HE (2009) Functional imaging of the parietal cortex during action execution and observation. Cereb Cortex 19:624-639.

Facchini S, Muellbacher W, Battaglia F, Boroojerdi B, Hallett M (2002) Focal enhancement of motor cortex excitability during motor imagery: a transcranial magnetic stimulation study. Acta Neurol Scand 105:146-151.

Fadiga L, Fogassi L, Pavesi G, Rizzolatti G (1995) Motor facilitation during action observation: a magnetic stimulation study. J Neurophysiol 73:26082611.

Fadiga L, Buccino G, Craighero L, Fogassi L, Gallese V, Pavesi G (1999) Corticospinal excitability is specifically modulated by motor imagery: a magnetic stimulation study. Neuropsychologia 37:147-158.

Gangitano M, Mottaghy FM, Pascual-Leone A (2001) Phase-specific modulation of cortical motor output during movement observation. Neuroreport 12:1489-1492.

Gangitano M, Mottaghy FM, Pascual-Leone A (2004) Modulation of premotor mirror neuron activity during observation of unpredictable grasping movements. Eur J Neurosci 20:2193-2202.

Hashimoto R, Rothwell JC (1999) Dynamic changes in corticospinal excitability during motor imagery. Exp Brain Res 125:75-81.

He SQ, Dum RP, Strick PL (1993) Topographic organization of corticospinal projections from the frontal lobe: motor areas on the lateral surface of the hemisphere. J Neurosci 13:952-980.

Izumi S, Findley TW, Ikai T, Andrews J, Daum M, Chino N (1995) Facilitatory effect of thinking about movement on motor-evoked potentials to transcranial magnetic stimulation of the brain. Am J Phys Med Rehabil 74:207-213.

Jeannerod M (2001) Neural simulation of action: a unifying mechanism for motor cognition. Neuroimage 14:S103-109.

Jenny AB, Inukai J (1983) Principles of motor organization of the monkey cervical spinal cord. J Neurosci 3:567-575.

Kasai T, Kawai S, Kawanishi M, Yahagi S (1997) Evidence for facilitation of motor evoked potentials (MEPs) induced by motor imagery. Brain Res 744:147-150.

Keizer K, Kuypers HG (1989) Distribution of corticospinal neurons with collaterals to the lower brain stem reticular formation in monkey (Macaca fascicularis). Exp Brain Res 74:311-318.

Kennedy C, Sakurada O, Shinohara M, Jehle J, Sokoloff L (1978) Local cerebral glucose utilization in the normal conscious macaque monkey. Ann Neurol 4:293-301.

Kiers L, Fernando B, Tomkins D (1997) Facilitatory effect of thinking about 
movement on magnetic motor-evoked potentials. Electroencephalogr Clin Neurophysiol 105:262-268.

Kraskov A, Dancause N, Quallo MM, Shepherd S, Lemon RN (2009) Corticospinal neurons in macaque ventral premotor cortex with mirror properties: a potential mechanism for action suppression? Neuron 64:922-930.

Kuypers HG (1981) Anatomy of the descending pathways. In: Handbook of physiology, section 1: the nervous system, motor control. (Brookhart JM, Mountcastle VB, eds), pp 597-666. Bethesda, MD: American Physiological Society.

Léonard G, Tremblay F (2007) Corticomotor facilitation associated with observation, imagery and imitation of hand actions: a comparative study in young and old adults. Exp Brain Res 177:167-175.

Li S, Kamper DG, Stevens JA, Rymer WZ (2004) The effect of motor imagery on spinal segmental excitability. J Neurosci 24:9674-9680.

Maeda F, Kleiner-Fisman G, Pascual-Leone A (2002) Motor facilitation while observing hand actions: specificity of the effect and role of observer's orientation. J Neurophysiol 87:1329-1335.

Maier MA, Perlmutter SI, Fetz EE (1998) Response patterns and force relations of monkey spinal interneurons during active wrist movement. J Neurophysiol 80:2495-2513.

Moll L, Kuypers HG (1977) Premotor cortical ablations in monkeys: contralateral changes in visually guided reaching behavior. Science 198: 317-319.

Montagna M, Cerri G, Borroni P, Baldissera F (2005) Excitability changes in human corticospinal projections to muscles moving hand and fingers while viewing a reaching and grasping action. Eur J Neurosci 22:15131520.

Moschovakis AK, Gregoriou GG, Savaki HE (2001) Functional imaging of the primate superior colliculus during saccades to visual targets. Nat Neurosci 4:1026-1031.

Patuzzo S, Fiaschi A, Manganotti P (2003) Modulation of motor cortex excitability in the left hemisphere during action observation: a single- and paired-pulse transcranial magnetic stimulation study of self- and nonself-action observation. Neuropsychologia 41:1272-1278.

Prut Y, Fetz EE (1999) Primate spinal interneurons show pre-movement instructed delay activity. Nature 401:590-594.

Prut Y, Perlmutter SI (2003) Firing properties of spinal interneurons during voluntary movement. I. State-dependent regularity of firing. J Neurosci 23:9600-9610.

Raos V, Evangeliou MN, Savaki HE (2004) Observation of action: grasping with the mind's hand. Neuroimage 23:193-201.

Raos V, Evangeliou MN, Savaki HE (2007) Mental simulation of action in the service of action perception. J Neurosci 27:12675-12683.

Riddle CN, Edgley SA, Baker SN (2009) Direct and indirect connections with upper limb motoneurons from the primate reticulospinal tract. J Neurosci 29:4993-4999.

Romani M, Cesari P, Urgesi C, Facchini S, Aglioti SM (2005) Motor facilitation of the human cortico-spinal system during observation of biomechanically impossible movements. Neuroimage 26:755-763.
Rossini PM, Rossi S, Pasqualetti P, Tecchio F (1999) Corticospinal excitability modulation to hand muscles during movement imagery. Cereb Cortex 9:161-167.

Savaki HE, Raos VC, Dermon CR (1992) Bilateral cerebral metabolic effects of pharmacological manipulation of the substantia nigra in the rat: unilateral intranigral application of the inhibitory GABAA receptor agonist muscimol. Neuroscience 50:781-794.

Savaki HE, Kennedy C, Sokoloff L, Mishkin M (1993) Visually guided reaching with the forelimb contralateral to a "blind" hemisphere: a metabolic mapping study in monkeys. J Neurosci 13:2772-2789.

Savaki HE, Raos VC, Dalezios Y (1997) Spatial cortical patterns of metabolic activity in monkeys performing a visually guided reaching task with one forelimb. Neuroscience 76:1007-1034.

Sawaguchi T, Yamane I, Kubota K (1996) Application of the GABA antagonist bicuculline to the premotor cortex reduces the ability to withhold reaching movements by well-trained monkeys in visually guided reaching task. J Neurophysiol 75:2150-2156.

Schmidlin E, Brochier T, Maier MA, Kirkwood PA, Lemon RN (2008) Pronounced reduction of digit motor responses evoked from macaque ventral premotor cortex after reversible inactivation of the primary motor cortex hand area. J Neurosci 28:5772-5783.

Schütz-Bosbach S, Mancini B, Aglioti SM, Haggard P (2006) Self and other in the human motor system. Curr Biol 16:1830-1834.

Shimazu H, Maier MA, Cerri G, Kirkwood PA, Lemon RN (2004) Macaque ventral premotor cortex exerts powerful facilitation of motor cortex outputs to upper limb motoneurons. J Neurosci 24:1200-1211.

Sokoloff L, Reivich M, Kennedy C, Des Rosiers MH, Patlak CS, Pettigrew KD, Sakurada O, Shinohara M (1977) The $\left[{ }^{14} \mathrm{C}\right]$ deoxyglucose method for the measurement of local cerebral glucose utilization: theory, procedure, and normal values in the conscious and anesthetized albino rat. J Neurochem 28:897-916.

Stamos AV, Evangeliou MN, Savaki HE, Raos V (2009) Involvement of the spinal cord in the inhibition of overt actions during action simulation. Program No. 307.9. 2009 Neuroscience Meeting Planner. Chicago, IL: Society for Neuroscience, 2009. Online.

Strafella AP, Paus T (2000) Modulation of cortical excitability during action observation: a transcranial magnetic stimulation study. Neuroreport 11:2289-2292.

Urgesi C, Candidi M, Fabbro F, Romani M, Aglioti SM (2006) Motor facilitation during action observation: topographic mapping of the target muscle and influence of the onlooker's posture. Eur J Neurosci 23: 2522-2530.

Yahagi S, Kasai T (1998) Facilitation of motor evoked potentials (MEPs) in first dorsal interosseous (FDI) muscle is dependent on different motor images. Electroencephalogr Clin Neurophysiol 109:409-417.

Yahagi S, Shimura K, Kasai T (1996) An increase in cortical excitability with no change in spinal excitability during motor imagery. Percept Mot Skills $83: 288-290$. 